\title{
X-Ray Vision: The Mental Genealogy of the Anatomy of the Interior of the Human Body
}

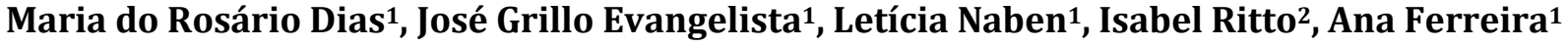 \\ ${ }^{1}$ CIMPS-EM-Campus Universitário, Quinta da Granja, Monte de Caparica, Caparica, Portugal \\ ${ }^{2}$ CIEBA-Faculdade de Belas-Artes, Universidade de Lisboa, Lisboa, Portugal \\ Email: mariadorosario.dias@gmail.com
}

How to cite this paper: do Rosário Dias, M., Evangelista, J.G., Naben, L., Ritto, I. and Ferreira, A. (2019) X-Ray Vision: The Mental Genealogy of the Anatomy of the Interior of the Human Body. Journal of Biosciences and Medicines, 7, 68-76. https://doi.org/10.4236/jbm.2019.78005

Received: July 10, 2019

Accepted: August 18, 2019

Published: August 21, 2019

Copyright $\odot 2019$ by author(s) and Scientific Research Publishing Inc. This work is licensed under the Creative Commons Attribution International License (CC BY 4.0).

http://creativecommons.org/licenses/by/4.0/

\begin{abstract}
Background: The concepts of the Young Person and of an Elderly Person go through an evolutionary diversification during the Human lifecycle and are permeable to diverse biopsychosocial factors involved in the definition of the lifecycle stages. Therefore, assessing this subjectivity in different stages of development and population fringes may be a determinant key factor to assist the implementation of successful disease prevention and health promotion strategies. Aim: The aim of the present study was to discern how Fine Arts' college students of the Anatomy class mentally represent the inner morphology of the human body of the Young Person and of an Elderly Person. Method: For such, 126 students were asked to draw the interior of the body of a Young Person and of an Elderly Person. In all, 252 drawings were collected and analysed, based on a coding data grid designed for this purpose. Hence, a comparative analysis of these two different vital lifecycle phases was achieved. Results: The results suggest anatomical differences in the pictorial representation of the Young Person and of the Elderly Person, namely in the accentuation of the curvatures of the vertebral column, retrusion of the lower jaw and muscle flaccidity. Conclusions: Although the body schema was the same for all individuals, the body image was singularly depicted and linked to each individual's history, representing a synthesis of his idiosyncratic perceptions, experiences and particularities.
\end{abstract}

\section{Keywords}

Mental Representation, Human Inner Body, Young Person, Elderly Person, Drawings

\section{Introduction}

The concepts of the Young Person and of the Elderly Person go through an evolutionary diversification during the lifecycle and are permeable to a panoply of 
factors involved in the definition of the lifecycle stages. The body image (imago) is not a physiologically static phenomenon but rather a complex and dynamic phenomenon that is acquired, formulated and structured in a continuous intraand inter-psychical contact with the World [1].

Previous studies developed by the Egas Moniz-Multidisciplinary Research Centre in Health Psychology [2] [3] have shown that the pictorial representations of a "Healthy Person" emerge as a form of projective identification, for the gender of the participant is consistent with that of the human figure they have portrayed. On the contrary, in the pictorial representations of "Sick Person", the gender of the figure depicted is the opposite of that of the participant who has sketched the drawing. This is evidence of the difficulty of participants to identify themselves with their mental representation of "Sick Person". Likewise, in a study conducted over the concept of health and illness in colorectal cancer patients [3], results showed that patients resorted to a projective identification mechanism which attested to a connection between being healthy and being young, whereas the "Sick Person" was often represented in rigid postures.

Furthermore, in a longitudinal study [4], it was inquired about the mental representations of the inside of the human body in undergraduate students of Health Sciences, an intriguing aspect occurred with regards to the representation of the skin, as it was not always depicted. Students were asked to draw a Healthy Person and a Sick Person before and after they were exposed to the contents of the curricular unit of Human Anatomy. Results showed that, in some drawings, the skin, as a boundary between the exterior and the interior of the human body, was absent. That absence occurred regardless of representation type (Healthy Person or Sick Person). Rather than interpreting this finding as a "broad-spectrum disorder" in self-object differentiation, we could interpret it as a symptom of a normal, yet defensive, dehumanization of the inner-body mental representation, which could serve the unconscious purpose of keeping a safe emotional distance from the notion of mortality.

Hence, this study aims to understand how Fine Arts undergraduate students who attend the curricular unit of Human Anatomy at a Higher Education Institute elaborate intra-psychically the mental representation of the internal morphology of the human body of a "Young Person" and of an "Elderly Person", resorting to pictorial drawings as a projective technique.

\section{Material and Methods}

A descriptive and longitudinal study of exploratory nature was conducted to satisfy the purposes of the present investigation's analytical model, which followed a qualitative-based methodological strategy (a content analysis of 266 drawings), combined with quantitative parameters.

\subsection{Participants}

The study was carried out in Portugal, at Fine Arts Higher Education Institute located in the Lisbon area. All participants were aged between 18 - 42 years and 
had not yet been introduced to the contents inherent to the curricular unit of Human Anatomy. The sample was collected in a classroom setting, where 133 students of both genders participated (31 men and 102 women).

\subsection{Data Collection}

The cognitive-emotional knowledge of the participants involved in the study regarding the human body was evaluated through drawings sketched at the beginning of the semester, as a projective instrument. This method of qualitative nature was used in previous studies [4] [5] in which in a population of children, adolescents and adults, subjects were asked to draw the interior of the human body. The results obtained suggested that, in general, these (pre)concepts are early acquired on the cognitive-emotional level.

In the present study, the participants were instructed to draw the interior of the Human body at discretion, in profile view. For this purpose, they were asked to complete an investigation protocol which was divided into two sections, section A and B. Section A called for the elaboration of two pictorial representations, one representing the interior of the body of a Young Person, and the other representing the interior of the body of an Elderly Person. In this section, participants were also instructed to characterize the human figures drawn according to age and gender. Drawings were captioned by researchers when the sample was collected in case of doubts concerning the drawings' peculiar representations. Also, in this section, participants were asked to answer two open-ended questions: 1) "What is a Young Person?" and 2) "What is an Elderly Person?". Section $\mathrm{B}$ requested the completion of a sociodemographic questionnaire and the answer to two additional questions: a) "Is this your first time attending the Anatomy Curriculum Unit?" and b) "Have you had any training in Human Anatomy?".

Subsequently, a pictorial content analysis grid was elaborated for the present study, in order to proceed with the analysis of 266 drawings collected. The grid encompassed ten main categories: 1) Gender, 2) Figure Drawing, 3) Age, 4) Anatomical Structure, 5) Skin; 6) Head; 7) Spinal Column; 8) Torso; 9) Accessories and 10) Injuries. In addition to these categories, additional analytical subcategories were developed to enhance the content analysis of the designed drawings: a) Gender of the sampled subject, b) Gender attributed to the drawing subject, c) Age of the sampled subject, d) Age attributed to the drawing subject, e) Height of the individual; f) Body image, g) Aging signs (signs, spots, wrinkles, warts and excess skin); h) Hair, i) Nose, j) Ears, k) Lower jaw (protrusion, retrusion and normal); 1) Cane.

\subsection{Methodological Procedures}

The data were collected on the first day of classes of Fine Arts' students enrolled in the curricular unit of Human Anatomy at a Higher Education Institute. Each participant received a research protocol where they were invited to draw with 
graphite pencil the internal morphology of the human body of a Young Person and of an Elderly Person. Once both drawings were completed, participants were invited to characterize the drawings they had made according to age and gender and to attribute a gender to the Human figure they had drawn, as well as to caption the components of the interior of the body drawn. Participants had $20 \mathrm{mi}-$ nutes to complete the task. All participants gave their consent to participate in the study. A total of 133 protocols were collected, which corresponded to 266 drawings. The drawings were then analysed through a content analysis grid created to evaluate the mental representation of the interior of the body of the Young Person and of the Elderly Person. The quantitative data were later analysed with the software SPSS (Statistical Package for the Social Sciences), version 19.0 for Windows. Qualitative data (i.e. open responses) in turn, were analysed through the content analysis of written narrative. The paper size for the data collection was an A4 sheet, which may have made the readability of some anatomical details difficult and may constitute a methodological limitation.

\section{Results and Discussion}

The results of the analysis of the two pictorial representations requested gave origin to two different profiles, the profile of the Young Person and the profile of the Elderly Person, distinguished by particular and antagonistic traits attributed to each pictorial representation.

\subsection{Profile of the Young Person}

The profile of the Young Person derived from the analysis of the mental representation of the Human inner body illustrated in the research protocols, which pointed to specific prominent characteristics, in opposition to the profile of the Elderly Person. Amongst them was the fact that $54.14 \%$ portraits were identified by the subjects as female in terms of gender. Furthermore, in the category Age, $36.8 \%$ portraits were 20 years old, which the predominant age group was attributed by the participants to the portraits drawn.

In the category Anatomical Structure, particularly in the subcategory Body Image, $66.9 \%$ portraits were pictorially represented with a normal body image. In the category Skin, $73.7 \%$ portraits were pictorially outlined by the skin organ, without showing any Aging Signs (e.g., signs, spots, wrinkles, warts and excess skin). In fact, the skin was represented with well-defined contours, denouncing positive pictorial investment (Figure 1(C)).

In the category Head, the subcategories Hair and Ears did not show significant change, whereas in the subcategory Nose, $39.8 \%$ portraits that pictorially represented a nose, did so drawing a normal-sized nose. Similarly, in the subcategory Lower Jaw, 71.4\% portraits had a normal lower jaw, with no vestigial retrusion or protrusion. Furthermore, $22.6 \%$ portraits had teeth represented (Figure 1(B)).

In the category Spinal Column, 97\% portraits were pictorially represented with a normal spine curvature (Figure $1(\mathrm{E})$ ). Interestingly, this feature was several 


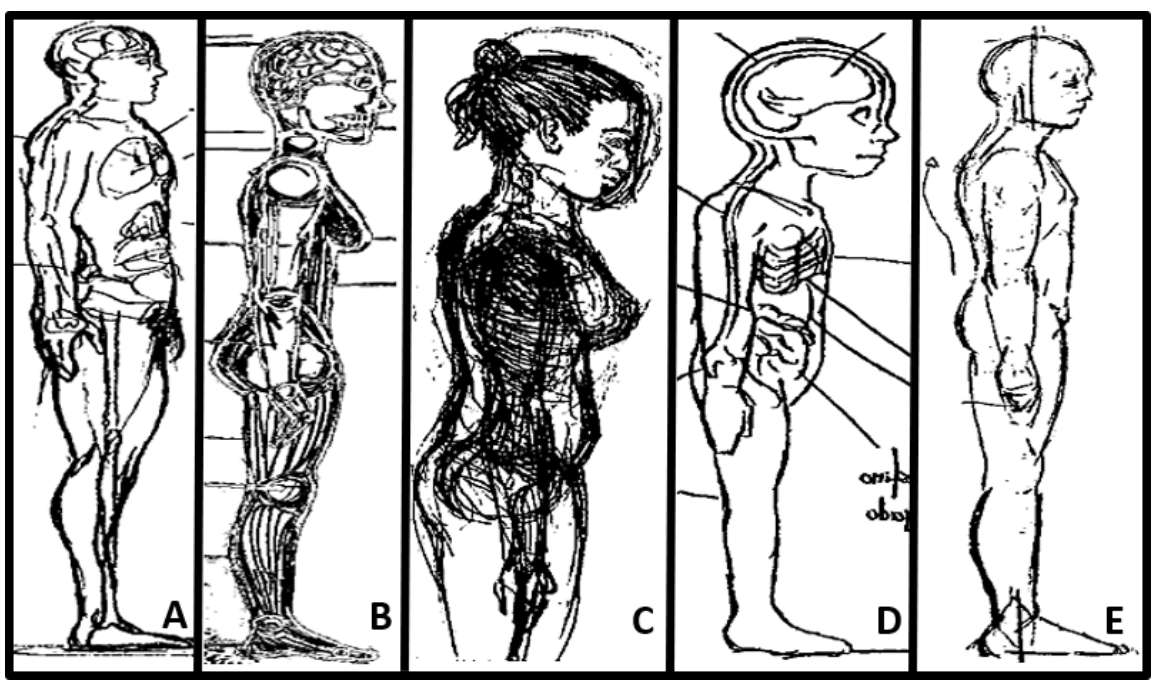

Figure 1. Examples of portraits drawn by subjects regarding the profile of a young person.

times emphasized in the portraits through arrows and signs, attesting to the importance and the specific intrinsic association of this trait to youthfulness. Likewise, in the category Torso, particularly in the subcategory Abdomen, 67.7\% portraits were pictorially represented with a normal abdomen, mainly with a demarked athletic anatomical structure (Figure 1(A)). In addition, in the subcategory Breasts, $33.8 \%$ portraits were pictorially represented with normal physiognomic contours, with as little as $1.5 \%$ being pictorially represented as saggy. No features were pictorially represented in the categories Accessories and Injuries, associated to the Young Person profile.

Regarding the qualitative answer analysis of the open-ended questions, the average age indicated to qualify a Young Person is 35 years (18.88\%). Other features attributed by the participants of the study to a Young Person included, in order of prevalence: 1) Energetic or active (18.03\%); 2) Healthy (16.31\%) and 3) Physically fit (14.16\%). The codes identified in the answers to the open-ended questions seem to corroborate the projected pictorial mental representation of the subjects in the portraits they have drawn, from which derived the profile of the Young Person of the present study (Figure 1(D)).

\subsection{Profile of the Elderly Person}

The profile of the Elderly Person also derived from the analysis of the mental representation of the Human inner body illustrated in the research protocols. In opposition to the profile of the Young Person, in the Elderly Person profile there is a decrease in the representation of the female gender, with $48.87 \%$ portraits identified as being female. Nevertheless, the female gender was the most represented gender in the Elderly Person profile.

In the category Age, the two age groups predominantly attributed to the portraits of the Elderly Person, ranged between 70 Years (17.3\%) and 80 Years (18.8\%). In the category Anatomical Structure, particularly in the subcategory 
Body Image, there was a significant increase of human figures with an obese body image in the portraits drawn (32.3\%), which is relevant when compared to the Young Person profile (1.5\%). In addition, portraits reproduced with a normal body image in the Elderly Person profile were about half those of the Young Person profile (38.3\%). In the category Skin, $71.4 \%$ portraits were pictorially outlined by the skin organ, with $27.1 \%$ showing Aging Signs (e.g., signs, spots, wrinkles, warts or excess skin). In fact, Wrinkles (15.8\%) and Excess Skin (6.8\%) were the most represented features of the subcategory Aging Signs in the drawings.

In the category Head, the subcategory Nose showed a significant increase in terms of nose size in the Elderly Person profile (18.8\%), when compared to the Young Person profile (8.3\%). Correspondingly, in the Elderly Person profile, there is a decrease in the representation of the subcategory Lower Jaw (51.1\%), with an emphasis on retrusion (19.5\%) and protrusion (15\%). Furthermore, in the subcategory Teeth, there was a positive increase of pictorial representation absence of teeth in the Elderly Person profile (91\%). This lack of representation may be associated with progressive edentulism experienced throughout the human lifecycle.

The category Spinal Column was the one that suffered a greater percentual change regarding the results obtained, when compared to the Young Person profile. In fact, the Spinal Column of the Elderly Profile was characterized as kyphosis (78.2\% against $1.5 \%$ in the Young Person profile) (Figure 2(D) and Figure 2(E)). This intrinsic characteristic of the Elderly Person profile, as well as the normal curvature in the Young Person profile, was again stressed in portraits through arrows and signs, attesting to the importance of this trait and its specific association to old age.

The category Torso confirms this shift in the mental representation of in the Elderly Person profile body image, particularly in the subcategory Abdomen. In fact, there is a significant decrease of human figures drawn with a normal abdomen in the Elderly Person profile (41.4\%) (Figure 2(A)), when compared to that of the Young Person profile (67.7\%). Moreover, in the Elderly Person profile, an abdominal prominence that resembles a marsupial pouch (Figure 2(B)) is highlighted in the portraits (26.3\%). In addition, in the subcategory Breasts, there was a significant increase of human figures represented with saggy breasts (35.3\%) (Figure 2(C)) when compared to the Young Person profile (1.5\%), attesting to the loss of muscle tone during the human lifecycle trajectory. Hence, the pictorial representation of the normal breasts decreased from $33.8 \%$ in the Young Person profile to 5.3\% in the Elderly Person profile.

In the category Accessories, two subcategories were represented-Glasses and Cane-with the latter being the most represented (12.8\%) (Figure 2(F)). The inclusion of these subcategories added to the Human figures highlighted the vulnerabilities and the bio-psycho-physiological limitations acquired with the aging process. In the category Injuries, we found injuries recorded in $16.4 \%$ portraits, with bone fractures as the most frequent injury. 


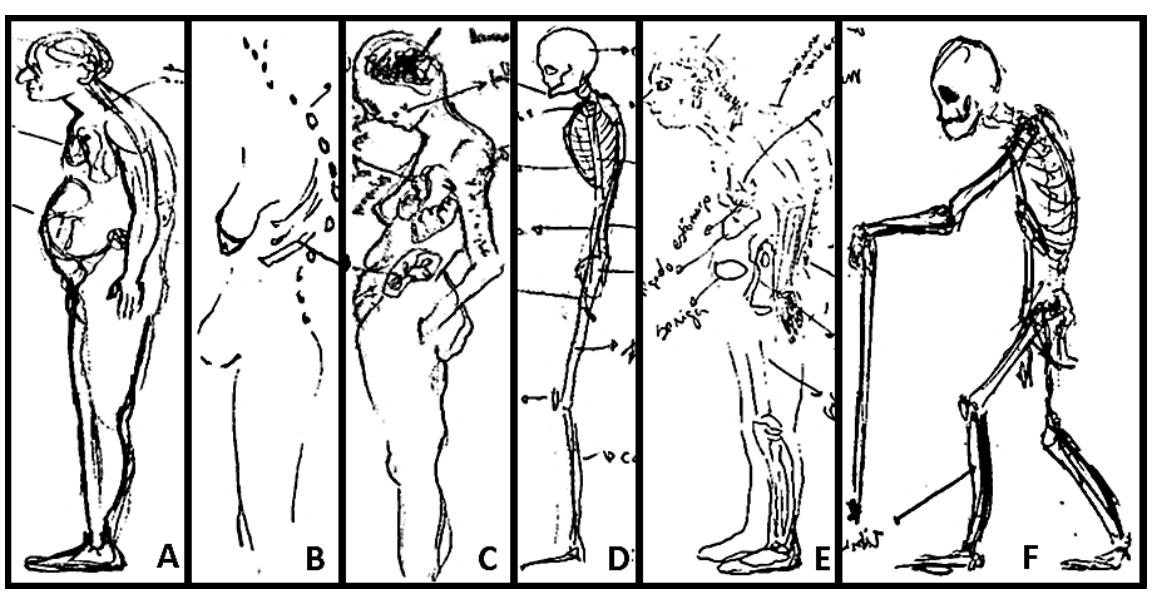

Figure 2. Examples of portraits drawn by subjects regarding the profile of an Elderly Person.

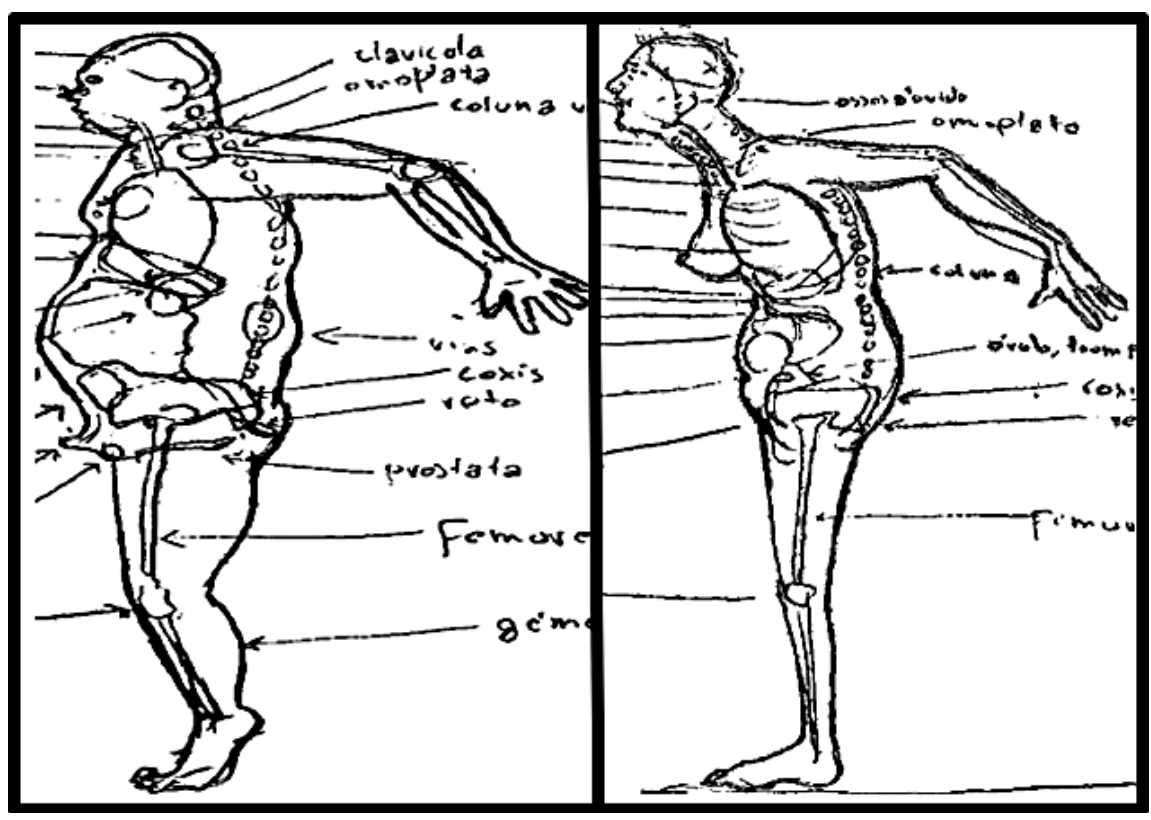

Figure 3. Examples of Human figure portraits representative of the peripessoal space.

On the other hand, concerning the body schema of the Human figures drawn, in the Elderly Person profile there was notorious movement (kinesthesia), associated with a very dynamic relational space (proxemics). The Elderly Person seemed, therefore, to expand their peripersonal space in an attempt to reduce distances related to the other people. In contrast, in portraits of the Young Person, it was noticed an inert and static body schema (Figure 3 ).

Regarding the qualitative answer analysis of the open-ended questions, the average age indicated to qualify an Elderly Person is 65 years (16.35\%). Other features attributed by the participants of the study to an Elderly Person included, in order of prevalence: 1) Fragile and or weak (14.07\%); 2) Mobility decline (11.79\%) and 3) Decreased bone and muscle structure (11.03\%). However, un- 
like what was observed in the Young Person profile, these indicators were not observable in the pictorial representations elaborated by the participants that gave origin to the Elderly Person profile. In contrast, indicators easily observed in portraits such as kyphosis (78.2\%) or saggy skin (35.3\%), were casually mentioned in the content analysis of the open answers (kyphosis $=9.13 \%$ and saggy skin $=3.80 \%)$.

\section{Conclusions}

The characteristics attributed to the analysed drawings depict two singular profiles: the profile of the Young Person and the profile of the Elderly Person. The anatomical differences between the profile of the Young Person and of the Elderly Person are mainly related to the accentuation of the curvatures of the vertebral column, a lower retrograde mandible, muscle flaccidity and wrinkles, traits rather related to the profile of the Elderly Person.

In some cases, the age attributed to the Young Person's drawings is on average very low, in sharp contrast to the age attributed to drawings of the Elderly Person, which is on average is higher. In fact, some mental representations of the Young Person's drawings seem to resemble those of infant, while the mental representations of the Elderly Person's drawings resemble those of real elders.

The use of accessories in the Profile of the Elderly Person (e.g. cane) reinforces the need to express the bio-psycho-physiological changes experienced with advancing age, in which reduced body mobility is the most preeminent feature in the drawings made by the participants.

Although the subjects were asked to reproduce the same task, the mental representation of each individual seems to be idiosyncratic in nature. However, all human beings need to support a paradigmatic (un)known (pre)concept: That both life and death come from the same place, the interior of the human body.

\section{Acknowledgements}

The authors of this project pay tribute to the research colleague Professor José Martins dos Santos, deceased to whom they dedicate the present article.

\section{Conflicts of Interest}

The authors declare no conflicts of interest regarding the publication of this paper.

\section{References}

[1] Schilder, P. (1994) A Imagem Do Corpo: As Energias Construtivas da Psique. Martins Fontes, São Paulo.

[2] Dias, M.R., Duque, A.F., Neves, A.C., Soares, F.A., Cardoso, J.I. and Carrão, L.M. (2006) Mente Sã em Corpo São. In: Pais Ribeiro, J.L., Leal, I. and Jesus, S., Eds., Minutes of the 6 th National Congress of Health Psychology, ISPA, Lisboa, 325-331.

[3] Dias, M.R. and Neves, A.C. (2016) Mente sã em Corpo São: Representação Mental Do Corpo Saudável e Doente na Criança. Omnia, 4, 55-64. 
[4] Dias, M.R., Neves, A.C., Simões, N.P., Evangelista, J.G. and Santos, J.M. (2016) X-Ray Vision: Mental Representation of the Human Body's Inner Morfology. International Journal of Current Research, 8, 32783-32791.

[5] Amann-Gainotti, M. and Antenore, C. (1990) Development of Internal Body Image from Childhood to Early Adolescence. Perceptual and Motor Skills, 71, 387-393. 